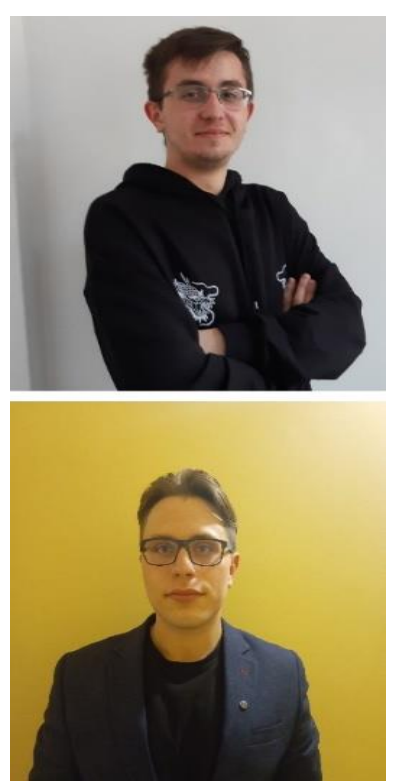

\section{РАЗРАБОТКА РЕГИСТРАТОРА СОПРОТИВЛЕНИЯ ИЗОЛЯЦИИ НА ОСНОВЕ МИКРОКОНТРОЛЛЕРА АРДУИНО}

\author{
А. П. Ляшенко, студент 2-го курса, \\ e-mail: lyashenko.andrey.02@mail.ru \\ ФГБОУ ВО «Калининградский государственный \\ технический университет» \\ М. Д. Кукуев, студент 2-го курса, \\ e-mail: misha.sev29@gmail.com \\ ФГБОУ ВО «Калининградский государственный \\ технический университет»
}

В работе представлены исследования, направленные на разработку регистратора сопротивления изоляции на основе микроконтроллера Ардуино, который использовался в сочетании с прибором контроля состояния изоляции ПКИ-2. Описаны особенности работы прибора ПКИ-2, выбран способ подключения микроконтроллера, разработана программа для него. Полученные результаты позволяют усовершенствовать процесс диагностирования изоляции электрооборудования, тем самым повысив его безопасность.

Ключевые слова: сопротивление изоляции, диагностирование, регистрация, Ардуино

\title{
ВВЕДЕНИЕ
}

Проблема повреждения изоляции актуальна как для высоковольтных, так и для низковольтных электросетей [1]. Последствия повреждения могут проявляться не только в выходе из строя одного из элементов электросети, но и в появлении экстратоков и перенапряжений, охватывающих значительную ее часть [2, 3]. Защита от повреждений изоляции может быть направлена и на преодоление последствий однофазного его повреждения [4], и на быстрое выявление дефекта [5]. Второе может быть реализовано на основе контроля за параметрами изоляции.

Одним из наиболее распространенных диагностических параметров изоляции является ее сопротивление. Контроль за сопротивлением изоляции в электросетях может осуществляться двумя способами [6]:

- при отсутствии рабочего напряжения;

- при наличии рабочего напряжения.

Второй способ является более информативным для решения задачи своевременного выявления дефектов изоляция, поскольку позволяет с меньшей периодичностью получать актуальные данные о состоянии оборудования. Это свойство приобретает все большее значение в последнее время в связи с расширением области применения диагностических методов, которые позволяют не только оценивать состояние изоляции, но и давать прогноз остаточного ресурса [7]. Реализация такого прогноза невозможна без постоянного отслеживания изменения величины ее сопротивления. 
Величина сопротивления твердой изоляции зависит от совокупности факторов:

- температура;

- влага;

- загрязнения;

- воздействующие перенапряжения.

Кроме того, сопротивление изоляции всей электросети зависит от количества ее элементов. Значение сопротивления может быть определено по следующему выражению:

$$
R=\left(\sum_{n} \frac{1}{R_{n}}\right)^{-1},
$$

где $R_{n}$ - сопротивление изоляции из $\mathrm{n}$ элементов.

В процессе эксплуатации электросети сопротивление изоляции существенно меняется. Это обусловлено как изменением количества элементов электросети в различных режимах работы электротехнического комплекса, так и изменением сопротивления изоляции отдельных элементов. Нормальные изменения сопротивления изоляции практически не связаны с возникновением в ней дефектов. Аварийные изменения обусловлены появлением какой-либо неисправности в элементах сети [8]. В связи с этим весьма важным является решение задачи измерения сопротивления изоляции с возможностью анализа причин изменения этой величины.

\section{ОБЪЕКТ ИССЛЕДОВАНИЯ}

Объектом исследования является регистратор изменений сопротивления изоляции, работающий в сочетании с известными устройства контроля состояния изоляции. Новизна работы обусловлена тем, что впервые предлагается система непрерывной регистрации ее величины.

\section{ЦЕЛЬ И ЗАДАЧИ ИССЛЕДОВАНИЯ}

Цель исследования заключалась в разработке регистратора сопротивления изоляции с возможностью сохранения результатов измерений. Для достижения этой цели решены следующие задачи:

- анализ работы устройства контроля состояния изоляции на примере прибора ПКИ-2;

- разработка дополнительного модуля регистрации сопротивления изоляции на основе Arduino UNO.

Актуальность поставленной цели обусловлена постоянным ростом роли электрооборудования в народном хозяйстве и жизнедеятельности людей. Поскольку одним из самых слабых мест любого электрооборудования является ее изоляция, то решаемые в исследовании задачи, направленные на повышение ее надежности и безопасности, представляются весьма важными.

\section{МЕТОДЫ ИССЛЕДОВАНИЯ}

Разработка регистратора заключалась в подготовке дополнительного модуля к известному устройству, измеряющему сопротивление изоляции. Таким устройством стал прибор ПКИ-2 [9]. Он предназначен для контроля сопротивления изоляции в трехфазной электросети переменного тока частотой 50 Гц и рассчитан на два напряжения контролируемой сети: 230 или $400 \mathrm{~B}$.

При снижении суммарного сопротивления изоляции ниже 16 кОм прибор запускает сигнализацию о повреждении. Прибор состоит из двух основных узлов: релейного блока и сигнального устройства. Схема релейного блока представлена на рис. 1.

Для контроля за состоянием электрической изоляции используется постоянный оперативный ток, получаемый от выпрямителя Д1...Д4. При нормальном состоянии изоляции 
через реле Р протекает постоянный ток, величина которого недостаточна для срабатывания реле.

При появлении утечки, вызванной повреждением изоляции, постоянный ток, протекающий через реле «Р», увеличивается и при критическом сопротивлении изоляции приводит к срабатыванию реле, замыкающему свой контакт в цепи питания сигнального устройства. Лампа «Л», сигнализирующая о недопустимом снижении сопротивления изоляции, в работе не использовалась. Визуальный контроль за состоянием изоляции электротехнической установки осуществляется с помощью килоомметра. Его показания регистрировались разработанным модулем.

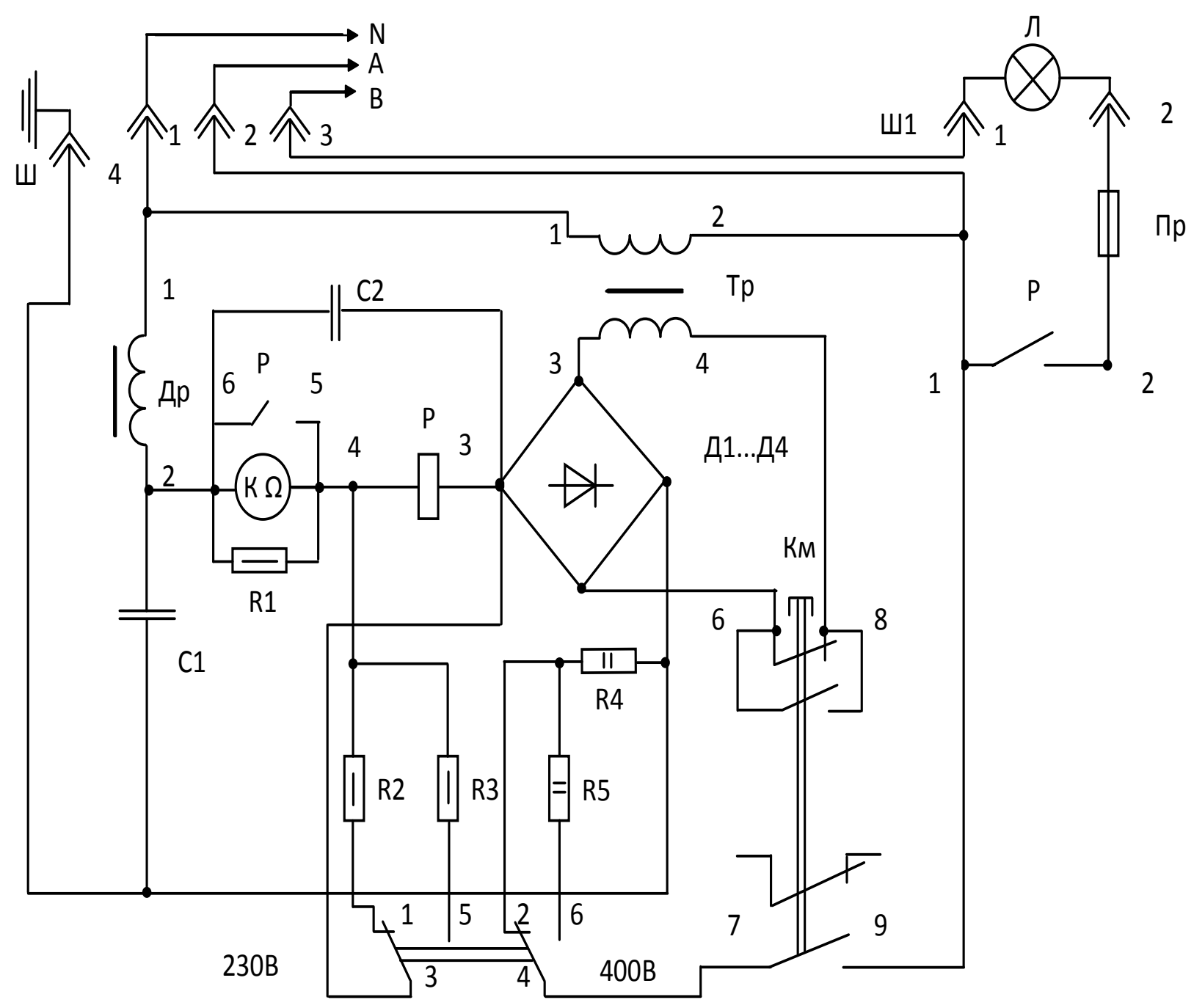

Рисунок 1 - Схема принципиальная ПКИ-2

Для анализа работы прибора контроля изоляции он был подключен к физической модели электросети напряжением 220B в соответствии с требованиями [9]. Для измерения тока, который протекает в аппарате, последовательно с килоомметром был включен измерительный шунт $\mathrm{R}_{\mathrm{m}}$. Предварительно при помощи осциллографа нами исследован сигнал, поступающий на килоомметр. Схема подключения осциллографа показана на рис. 2.

С помощью осциллографа установлена форма тока, протекающего через килоомметр. Зарегистрированная осциллограмма показана на рис. 3.

Как видно из рис. 3, через килоомметр протекает пульсирующий ток, что было учтено при разработке программы для регистрирующего модуля. Его подключение к прибору 
контроля состояния изоляции было осуществлено аналогично подключению осциллографа. Схема подключения приведена на рис. 4.

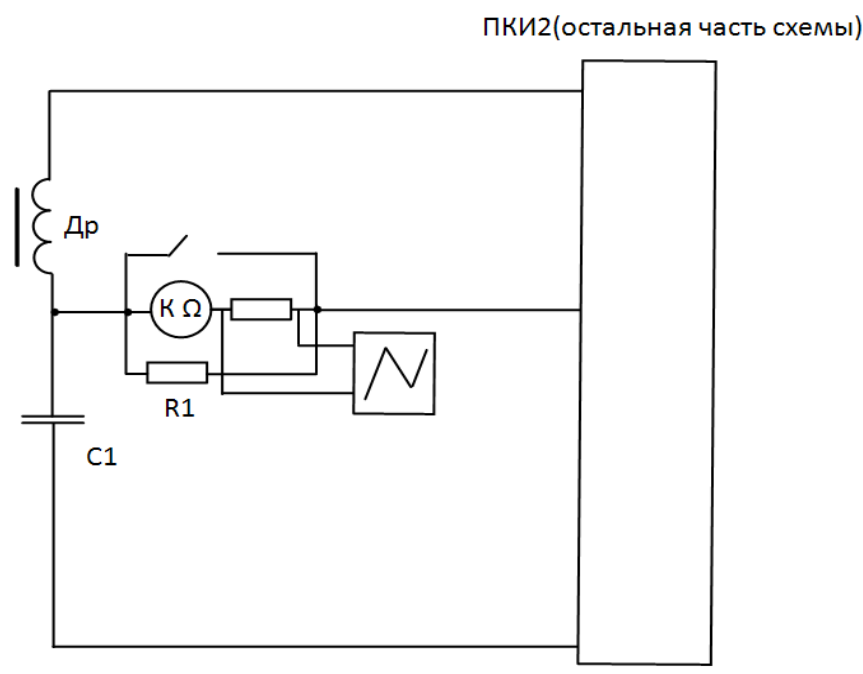

Рисунок 2 - Схема подключения осциллографа к прибору ПКИ-2

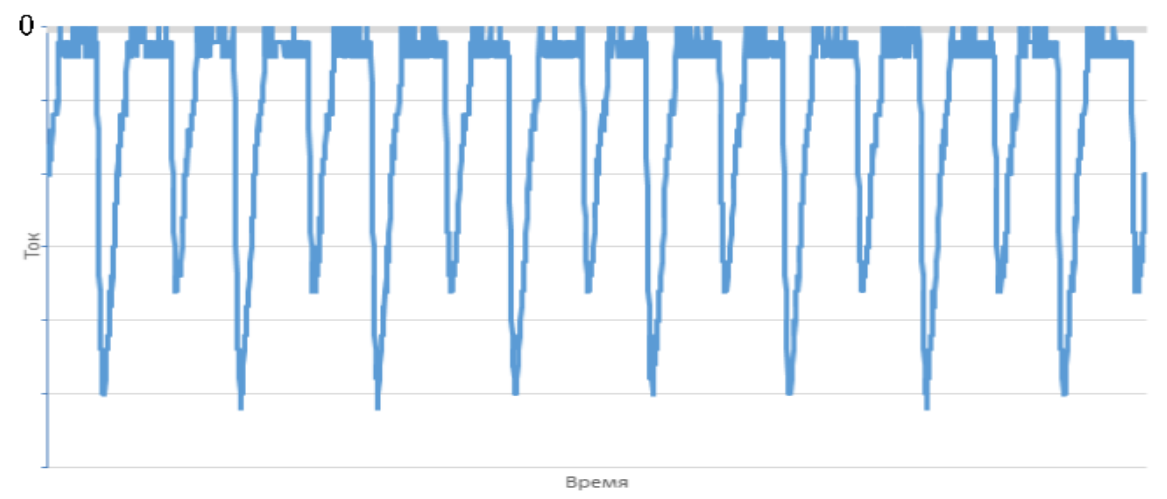

Рисунок 3 - График зависимости тока от времени

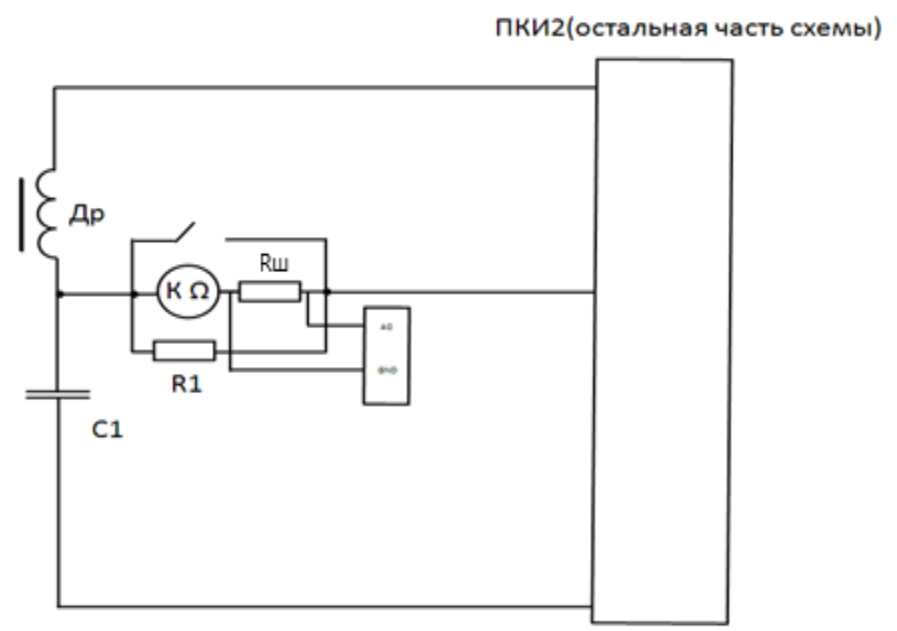

Рисунок 4 - Схема подключения микроконтроллера к ПКИ-2 через измерительный шунт 
Как видно из рис. 4, подключение микроконтроллера Arduino UNO осуществлено через измерительный шунт $\mathrm{R}_{ш}$. Напряжение измерительного шунта, пропорциональное току килоомметра, подавалось на вход микроконтроллера, осуществляющего обработку сигнала. Величина этого сигнала кратна значению сопротивления изоляции.

\section{РЕЗУЛЬТАТЫ ИССЛЕДОВАНИЯ}

Обработанные Arduino UNO значения выводились на экран ноутбука. Изображение собранной лабораторной установки, включающей модель электросети с возможностью управления сопротивлением изоляции, прибор ПКИ-2, ноутбук и микроконтроллер Arduino UNO, приведено на рис. 5. Алгоритм программы микроконтроллера показан на рис. 6.

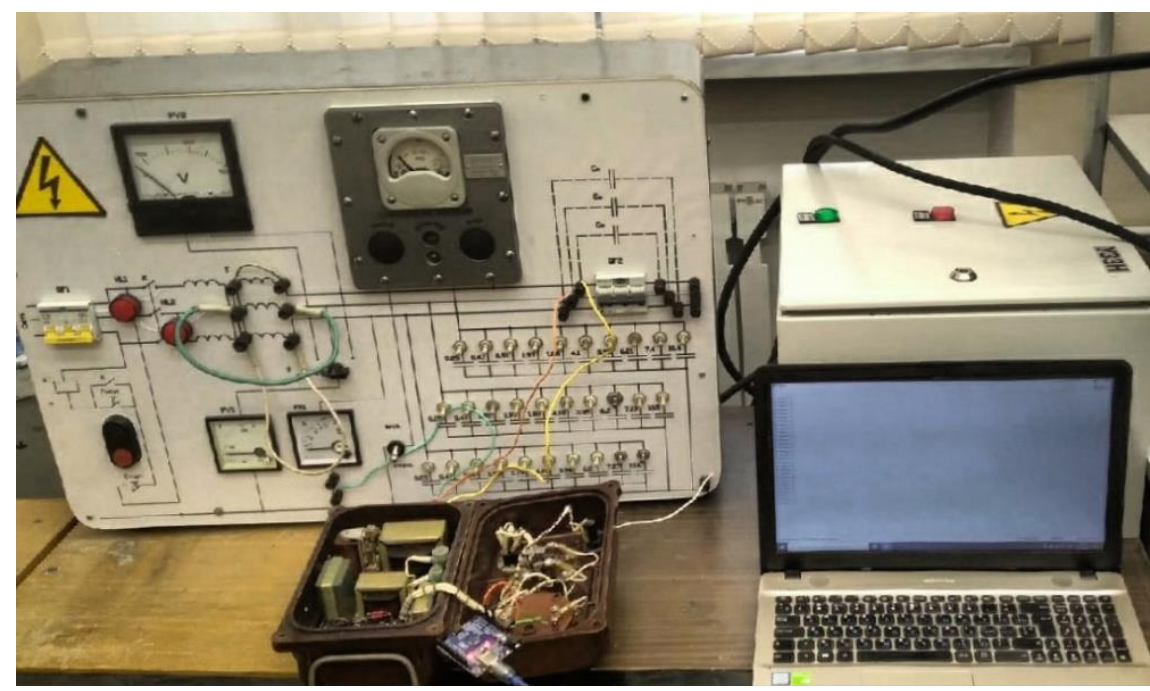

Рисунок 5 -Экспериментальная установка

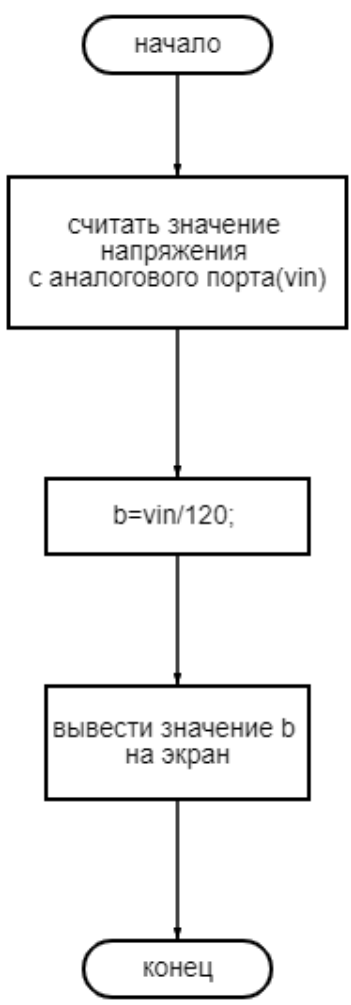

Рисунок 6 - Блок схема работы системной платы 
Программа для измерения напряжения с помощью Arduino UNO основана на возможности аналоговых входов системной платы измерять напряжение в диапазоне 0-5 В. Для этого использовался аналоговый вход под номером А0. Как только на него поступает напряжение, оно приобретает определенное цифровое значение. Однако его требуется перевести в омы, для этого был экспериментально подобран коэффициент пропорциональности. Полученное значение сопротивления изоляции выводится на экран компьютера с интервалом в одну секунду.

Значимость полученных результатов заключается в выявлении особенностей и основных подходов к регистрации сопротивления изоляции, а также в разработке программно-аппаратного комплекса для реализации этого.

\section{ЗАКЛЮЧЕНИЕ}

Разработанный модуль позволяет считывать показания прибора контроля состояния изоляции ПКИ-2. Их совместная работа дает возможность осуществлять регистрацию и накапливание значений сопротивления изоляции, тем самым выявляя опасные снижения этой величины, связанные с возникновением дефекта. Основным практическим результатом исследования стали результаты анализа возможности подключения модуля к устройству контроля изоляции. Теоретическим результатом работы можно считать разработку алгоритма программы для регистрации сопротивления изоляции.

\section{СПИСОК ЛИТЕРАТУРЫ}

1. Благинин, В. А. Резистивное заземление нейтралей в судовых электроэнергетических системах / В. А. Благинин, И. Е. Кажекин // Известия КГТУ. - 2010. № 17. - С.138-140.

2. Кажекин, И. Е. Описание процессов при дуговых однофазных замыканиях в низковольтных судовых электросетях с компенсированной нейтралью / И. Е. Кажекин // Морские интеллектуальные технологии. - 2019. - Т. 4, № 4 (46). - С. 83-87.

3.Кажекин, И. Е. Формирование максимальных перенапряжений при возникновении феррорезонансных процессов во время дуговых однофазных замыканий в низковольтных судовых электросистемах / И. Е. Кажекин // Вестник Астраханского государственного технического университета. Серия: Морская техника и технология. - 2020. - № 1. C. 115-124.

4. Кажекин, И. Е. Определение требований к защите судовых электроэнергетических систем от однофазных замыканий / И. Е. Кажекин // Электрика. - Москва, 2011. - С. 35-39.

5. Жерновой, Д. А. Автоматизация контроля состояния и поиска повреждений изоляции в распределительной сети систем электроснабжения с изолированной нейтралью / Д. А. Жерновой, Г. А. Сухарь, А. А. Тишков // Проблемы технического обеспечения войск в современных условиях: труды научно-практической конференции. - Санкт-Петербург: Военная академия связи, 2016. - С. 255-259.

6. Классификация и анализ способов контроля состояния изоляции в сетях напряжением до 1000 В / А. Б. Утегулов, Ж. А. Юсупов, Е. Т. Шахман [и др.] // Наука и техника Казахстана. - 2005. - № 2. - С. 153-158.

7. Иванов, Е. А. Методы контроля изоляции судовых электроэнергетических систем: учебное пособие / Е. А. Иванов, С. Е. Кузнецов. - Санкт-Петербург: Элмор, 1999. - 80 с.

8. Бирюлин, В. И. Автоматизация контроля состояния изоляции кабельных линий / В. И. Бирюлин, Д. В. Куделина, О. А. Камаева // Актуальные проблемы электроэнергетики: сборник научно-технических статей, посвящается 80-летию со дня рождения проф. С. В. Хватова. - Нижний Новгород: Нижегородский государственный технический университет им. Р. Е. Алексеева; Образовательно-научный институт электроэнергетики. 2018. - C. 262-266.

9. Прибор постоянного контроля изоляции ПКИ-2. Паспорт. - 13 с. 


\title{
DEVELOPMENT OF THE INSULATION RESISTANCE RECORDER \\ BASED ON THE ARDUINO MICROCONTROLLER
}

\author{
A. P. Lyashenko, student, \\ e-mail: lyashenko.andrey.02@mail.ru \\ Kaliningrad State Technical University \\ M. D. Kukuev, student, \\ e-mail: misha.sev29@gmail.com \\ Kaliningrad State Technical University
}

The paper presents research aimed at developing an insulation resistance recorder. The development was carried out on the basis of the Arduino microcontroller, which was used in combination with the PKI-2 insulation condition monitoring device. The article describes the features of the PKI-2 device operation, the method of connecting the microcontroller has been chosen, a program for it has been developed. The results obtained make it possible to improve the process of diagnosing the insulation of electrical equipment, thereby increasing its safety.

Key words: insulation resistance, diagnostics, registration, Arduino 